STEM CELLS

\section{Stem cells tackle diabetes}

Stem cells may be useful for treating type 2 diabetes, according to a study in mice.

Insulin-producing cells derived from human embryonic stem cells reduce blood sugar levels in mice with type 1 diabetes, but it was unclear whether the approach would work for type 2 diabetes, which is much more common in humans. To model the disease, Timothy Kieffer of the University of British Columbia in Vancouver, Canada, and his colleagues fed mice a high-fat diet. After five days, the mice had high blood glucose levels, and by seven weeks they exhibited other symptoms of type 2 diabetes.

Transplanting pancreatic cells grown from human embryonic stem cells into these mice increased their sensitivity to insulin. When combined with diabetes drugs, the transplants also reduced obesity and blood sugar levels. Stem Cell Rep. http://dx.doi.org/ 10.1016/j.stemcr.2015.02.011 (2015)

\section{CANCER}

\section{Leukaemia cells made normal}

Cancerous white blood cells from people with a form of leukaemia have been reprogrammed into immune cells that do not cause the disease in animals.

Immature immune cells called B cells cannot develop fully in people with precursor B-cell acute lymphoblastic leukaemia (B-ALL). Ravindra Majeti and his colleagues at Stanford University in California isolated diseased cells with specific mutations from 12 patients with B-ALL.

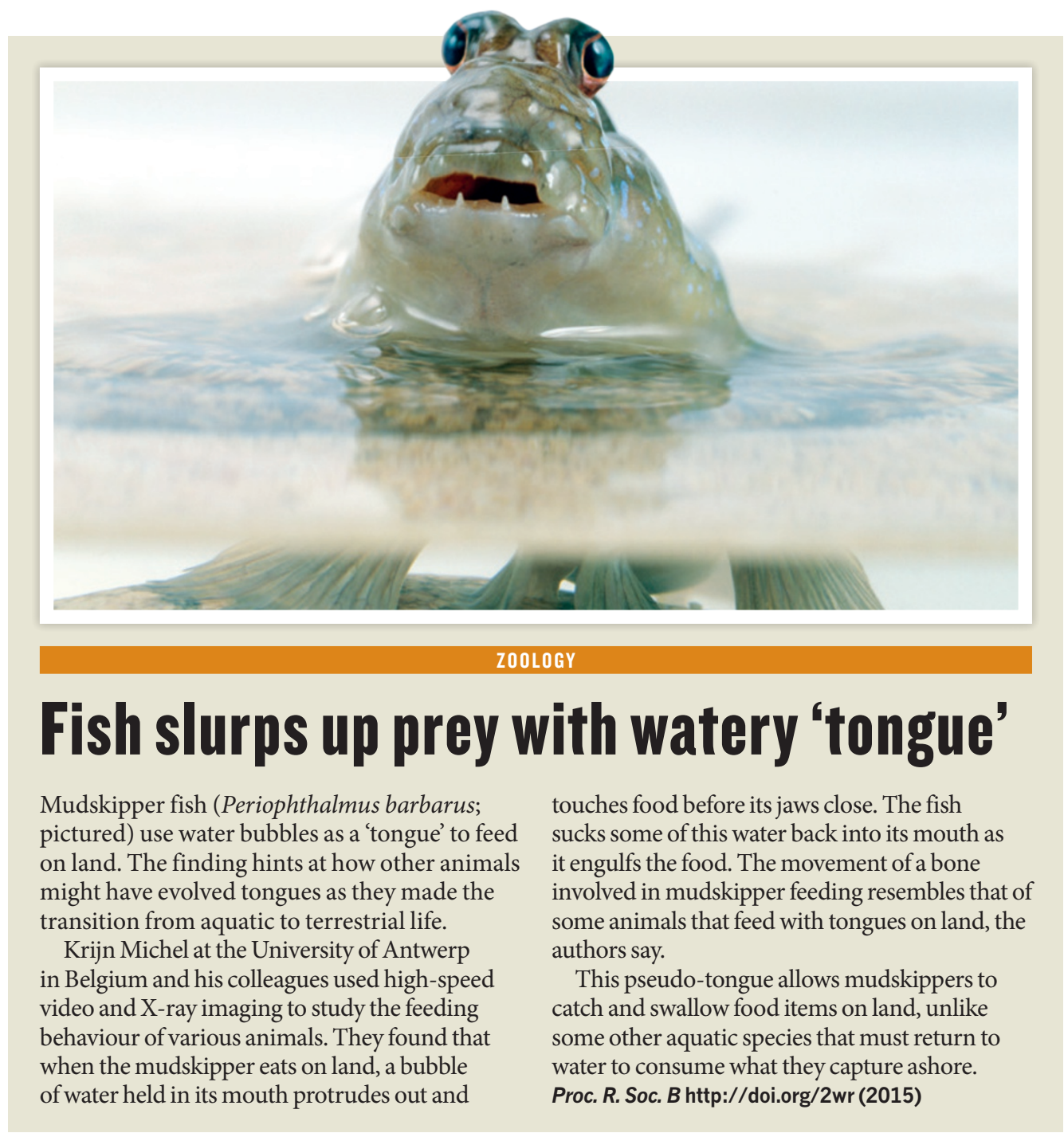

The researchers reprogrammed the cells by culturing them with molecules that promote the development of myeloid cells, which include several types of white blood cell. They ended up with cells that were similar to macrophages, which engulf pathogens, and did not develop into cancer when transplanted into mice. Turning on a myeloid regulatory gene in the cells achieved the same result.

This could be a therapeutic strategy for some people with B-ALL, the authors say. Proc. Natl Acad. Sci. USA http://doi.org/24t (2015)

\section{CHEMISTRV}

\section{Imaging of excited electron orbitals}

A technique could pave the way for imaging electron behaviour as chemical reactions happen.

Many reactions are governed by the behaviour of electrons in excited orbital states, but these states are difficult to capture because they last only a few picoseconds ( $10^{-12}$ seconds). Now, Masahiko Takahashi at Tohoku University in Sendai, Japan, and his colleagues have measured the excited states of molecules of acetone gas by probing them with ultrashort pulses of electrons.

Electrons in the pulse knocked excited electrons out of the atoms comprising the acetone. By simultaneously measuring the energy of the ejected electrons and those from the pulse, the team determined the energy and momentum of the molecule's excited state, which lasted for just 13.5 picoseconds.

Although the data quality is currently low, the results show that such measurements are feasible, say the authors. Phys. Rev. Lett. 114, 103005 (2015) 